\section{Unintentional secondary exogenous corticosteroid exposure and central serous chorioretinopathy}

\begin{abstract}
Purpose The purpose of this study is to describe a possible association between unintentional secondary exogenous corticosteroid exposure and central serous chorioretinpathy (CSCR).

Methods A retrospective review of three patients diagnosed with CSCR in one or both eyes and a history of possible unintentional secondary corticosteroid exposure. Clinical history, exam findings, optical coherence tomography, fluorescein angiography, and clinical course were reviewed.

Results Three patients, who did not use corticosteroids, reported suspected unintentional secondary corticosteroid exposure from living with family members or partners using dermatologic corticosteroid cream and/or inhalers frequently. Two of the three patients had a history of recurrent CSCR, one patient involving both eyes. After taking precautions to avoid secondary corticosteroid exposure, all three patients had complete resolution of CSCR without further recurrence during follow-up ranging from 2 to 4 years. Conclusions Patients with CSCR should be queried for both primary and possible unintentional secondary routes of corticosteroid exposure, which may contribute to this condition.
\end{abstract}

Eye (2015) 29, 577-579; doi:10.1038/ eye.2014.328; published online 16 January 2015

\section{Introduction}

Central serous chorioretinpathy (CSCR) is an idiopathic condition characterized by exudative detachment of the neurosensory retina. ${ }^{1}$ The pathophysiology is not completely understood but numerous associations have been proposed, with corticosteroid use being the most consistently reported risk factor. ${ }^{2-4}$ This report describes a possible association between CSCR and unintentional corticosteroid exposure from the environment (secondary exposure, SE), a new mode of exposure not previously described.

\section{Methods}

This is a retrospective review of the medical records of three patients diagnosed with CSCR at the University of California Davis Eye Center with history of unintentional SE to corticosteroids. All applicable institutional and governmental regulations concerning the ethical use of human volunteers were followed.

Clinical history, examination findings, optical coherence tomography (OCT), and fluorescein angiography (FA) were reviewed. The criteria for diagnosing a patient with CSCR included the biomicroscopical appearance of subretinal fluid (SRF), presence of PEDs, irregular retinal pigment epithelium (RPE) mottling, and FA appearance of either a focal leak, smokestack leak, or multifocal leak with staining. ${ }^{1}$ Primary exposure (PE) was defined as corticosteroids used by the patient. Unintentional SE was defined as history of the patient being in close physical contact with an individual (usually a family member or partner) known to use exogenous corticosteroids regularly.

\section{Case Reports}

Case 1 A 34-year-old male with a history of hypertension and hyperlipidemia presented with a second episode of CSCR in both eyes over 3 months. He worked as a medical
Department of

Ophthalmology and Vision Science, University of

California Davis Eye Center, Sacramento, CA, USA

Correspondence: SS Park, Department of Ophthalmology and Vision Science, University of California Davis Eye Center, 4860 Y Street, Suite 2400, Sacramento, CA 95817, USA;

Tel: +1 916734 6074;

Fax: +1 9167346197 .

E-mail: susanna.park@

ucdmc.ucdavis.edu

Received: 17 September 2014

Accepted: 7 December 2014

Published online:

16 January 2015

Meeting Presentation: Poster presentation at the Association for Research in Vision and Ophthalmology (ARVO) annual meeting, Fort Lauderdale, FL on May 6th, 2012. 
laboratory scientist and was under stress during his initial episode, but the etiology of the second episode was unclear. He denied a history of PE to corticosteroids, but reported just moving in with his girlfriend 6 months earlier who was using dermatologic hydrocortisone cream frequently. There was a concern for possible SE; after taking precautions to minimize this exposure, the CSCR resolved without any recurrences during the 4-year follow-up.

Case 2 A 47-year-old male with a history of hyperlipidemia presented with a third episode of CSCR in the left eye over 1 year. He admitted to being under stress from stock market investments for his first two episodes, but the etiology of his most recent episode was unclear. He denied PE to corticosteroids, but reported that his wife and son were both using dermatologic corticosteroid creams regularly. He occasionally helped his son apply the corticosteroid cream. After taking appropriate precautions, complete resolution of CSCR activity was noted with no further recurrences over the 3-year follow-up.

Case 3 A 40-year-old male with no significant past medical history presented with his first episode of CSCR in the left eye. He worked as a computer engineer and admitted to being under significant stress. He denied PE to corticosteroid, but lived with his daughter who used dermatologic corticosteroid creams for eczema and steroid inhalers for asthma on a regular basis. After taking precautions to minimize SE to corticosteroid, there was complete resolution of CSCR with no further recurrences over the 2-year follow-up.

\section{Discussion}

To our knowledge, this is the first report describing a possible association between CSCR and SE to corticosteroids. All three of our patients took precautions to minimize corticosteroid exposure, including ensuring that family members or partners washed their hands after corticosteroid application, avoiding direct application of the corticosteroid cream for family members, and minimizing close physical contact with family members or partners immediately after corticosteroid use. All three patients had complete resolution of CSCR with no recurrences during a follow-up period of 2-4 years.

The pathophysiology of CSCR is not completely understood. The choroid is thickened, and the resultant increase in choroidal hydrostatic pressure is thought to perhaps overwhelm the RPE and result in the accumulation of SRF and PEDs. ${ }^{3,5,6}$ The pathophysiologic role of corticosteroids in the exacerbation of CSCR may be via their action on the RPE, Bruch's membrane, or the choroid, but the exact mechanism remains unclear and requires further study. ${ }^{2,7-9}$
There are multiple reports describing the association between exogenous corticosteroid and CSCR. ${ }^{2-4}$ However, the possible association between CSCR and SE to corticosteroids is a new observation that warrants further investigation. Although this small case series does not prove a causal relationship, it highlights a possible association that may have been previously overlooked. In evaluating patients with CSCR, it may be important to inquire about both $\mathrm{PE}$ and $\mathrm{SE}$ to corticosteroids, especially among patients with unexplained recurrent or chronic CSCR. Corticosteroid use should be avoided when medically feasible, and those with possible SE should be advised to take precautions to minimize such exposure.

\section{Summary}

What was known before

- Exogenous exposure to corticosteroids is a risk factor for the development of CSCR.

What this study adds

- Unintentional secondary corticosteroid exposure from the environment (from living with family members or partners using frequent dermatologic corticosteroid cream and/or inhalers) is an important entity to consider in patients with unexplained recurrent CSCR.

- Precautions taken to minimize unintentional secondary corticosteroid exposure resulted in complete resolution of CSCR disease activity with no further recurrences in all patients.

\section{Conflict of interest}

The authors declare no conflict of interest.

\section{Acknowledgements}

This work was supported in part by an unrestricted grant from Research to Prevent Blindness New York, NY, USA to the University of California Davis Eye Center. Research to Prevent Blindness had no role in the study design, in the collection, analysis, and interpretation of the data; in the writing of the manuscript; or in the decision to submit the manuscript for publication.

\section{References}

1 Gemenetzi M, De Salvo G, Lotery AJ. Central serous chorioretinopathy: an update on pathogenesis and treatment. Eye 2010; 24: 1743-1756.

2 Bouzas EA, Karadimas P, Pournaras CJ. Central serous chorioretinopathy and glucocorticoids. Surv Ophthalmol 2002; 47: 431-448.

3 Nicholson B, Noble J, Forooghian F, Meyerle C. Central serous chorioretinopathy: update on pathophysiology and treatment. Surv Ophthalmol 2013; 58: 103-126. 
4 Haimovici R, Koh S, Gagnon DR, Lehrfeld T, Wellik S. Risk factors for central serous chorioretinopathy: a case-control study. Ophthalmology 2004; 111: 244-249.

5 Yannuzzi LA. Central serous chorioretinopathy: a personal perspective. Am J Ophthalmol 2010; 149: 361-363.

6 Goldstein BG, Pavan PR. 'Blow-outs' in the retinal pigment epithelium. Br J Ophthalmol 1987; 71: 676-681.
7 Arndt C, Sari A, Ferre M, Parrat E, Courtas D, De Seze J et al. Electrophysiological effects of corticosteroids on the retinal pigment epithelium. Invest Ophthalmol Vis Sci 2001; 42: 472-475.

8 Oikarinen AI, Uitto J, Oikarinen J. Glucocorticoid action on connective tissue: from molecular mechanisms to clinical practice. Med Biol 1986; 64: 221-230.

9 Yang S, Zhang L. Glucocorticoids and vascular reactivity. Curr Vasc Pharmacol 2004; 2: 1-12. 